\title{
Minimum Distance-Based Limited-Feedback Precoder for MIMO Spatial Multiplexing Systems
}

\author{
Alireza Ghaderipoor and Chintha Tellambura \\ Department of Electrical and Computer Engineering \\ University of Alberta, CANADA \\ Email: \{ghaderi, chintha\}@ece.ualberta.ca
}

\begin{abstract}
Precoding is a well-known method to reach the promised performance and capacity of multiple-input multipleoutput (MIMO) systems. Recent investigations, when the transmitter has the channel-state information (CSI), have revealed several precoding techniques. Minimum distance based precoders outperform precoders based on other criteria such as maximizing signal-to-noise ratio (SNR), minimizing the mean square error and maximizing the minimum singular value of the equivalent channel. On the other hand, when the CSI is not available at the transmitter, one resorts to limited feedback precoding methods. Previously, unitary matrices for precoding have been derived from subspace packing in the Grassmann manifold. In this paper, we use the same set of unitary matrices and enhance them by defining the precoder matrix to have a general form not unitary only. We extract the precoding parameters by applying the minimum-distance approach. Although in this case the number of feedback parameters is increased, the performance results are accordingly impressive. The optimality of quantization of feedback parameters is also presented.
\end{abstract}

\section{INTRODUCTION}

Multiple-input multiple-output (MIMO) systems increase the capacity of communication systems significantly [1]. Primary schemes for exploiting multiple antennas at the transmitter and/or receiver are based on maximizing the capacity by spatial multiplexing [2], or increasing the diversity order by space-time coding [3]. A common assumption on the design of space-time codes is that due to the availability of pilots or training sequences, the channel gains are known to the receiver but not to the transmitter. However, in communication systems that experience a slow fading environment, complete or partial knowledge of the channel information is available at the transmitter.

One way to exploit the channel information at the transmitter is precoding. The optimum precoder can be obtained if full channel state information (CSI) is available at the transmitter, since this allows the transmitted signal to be formed based on the eigen structure of the channel matrix [4]. There are several criteria that have been used for MIMO system optimization and lead to different precoding techniques. One criterion is the well-known water-filling solution, which aims to maximize the output capacity. Other designs using the weighted minimum mean square error (MMSE) criterion and the maximization of the minimum singular value of the channel matrix have been proposed in [5]. The optimal precoding scheme proposed in [6] is based on maximizing the minimum receive Euclidean distance and is shown to outperform the precoders designed based on other criteria, e.g. [7].

Due to the bandwidth constraints on the feedback channel, however, full CSI is not available at the transmitter. Therefore, precoding techniques using limited feedback of CSI are of interest [8]. Reference [8] designs a set of suboptimum unitary matrices for precoding. Depending on the receiver, optimal codebook designs are shown to be subspace packings in the Grassmann manifold using either the projection two-norm or the Fubini-Study distance. The codebook is known to both transmitter and receiver and the only feedback parameter is the index of the appropriate matrix.

In this paper, we propose to use a general structure precoder rather than a unitary one, for limited feedback systems, to improve the performance of codebooks proposed in [8]. We use the minimum distance-based method presented in [6] (for complete CSI case) to obtain the design parameters. The proposed scheme needs more feedback parameters than previous work, but the system performance is improved. Since the new feedback parameters are continuous, appropriate quantization is also discussed.

\section{SySTEM MODEL}

In a narrow-band, flat fading, multiple antenna system with $N_{t}$ transmit and $N_{r}$ receive antennas, when there are $M$ data substreams $\left(M \leqslant \min \left(N_{t}, N_{r}\right)\right)$ that are mapped to a signal constellation with unit average energy, and then precoded to be sent over the channel, the received vector will be

$$
\mathbf{r}=\sqrt{\frac{\rho}{M}} \mathbf{H F} \mathbf{s}+\mathbf{v}
$$

where $\mathbf{r} \in \mathcal{C}^{N_{r}}$ is the complex received vector, $\mathbf{s} \in \mathcal{C}^{M}$ is the transmitted vector, $\mathbf{v} \in \mathcal{C}^{N_{r}}$ is the additive noise vector $\mathbf{H} \in \mathcal{C}^{N_{r} \times N_{t}}$ is the channel matrix and $\mathbf{F} \in \mathcal{C}^{N_{t} \times M}$ is the precoder matrix.

We assume all elements in $\mathbf{v}$ and $\mathbf{H}$ are independent and identically distributed with complex Gaussian distribution, with zero mean and unit variance, which guarantees $\rho$ to be the received signal-to-noise ratio (SNR) for each transmitted signal. The factor $\frac{1}{\sqrt{M}}$ also assures that the total transmitted power in each channel use is $\rho$. We assume that $\mathbf{H}$ is changed after each transmission. For each channel realization, the precoder matrix $\mathbf{F}$ is constructed based on the feedback 
parameters, received from an error-free zero-delay feedback channel.

In this paper, $(\cdot)^{*},\|\cdot\|, E_{x}\{\cdot\}$ and $[\cdot]$ denote the Hermitian (transpose-conjugate), Frobenius norm, expectation over $x$, and ceil function, respectively. $\bar{b}$ represents $\sqrt{1-b^{2}}$ for the scalar $b$. All singular value decompositions are decreasingly ordered on the diagonal part. $\mathbf{U}$ is a unitary matrix if $\mathbf{U}^{*} \mathbf{U}=\mathbf{I}$ and $\mathbf{I}$ denotes the identity matrix.

\section{UNITARY PRECODING}

By singular value decomposition (svd) of the channel matrix, $\mathbf{H}=\mathbf{V}_{L} \boldsymbol{\Sigma} \mathbf{V}_{R}^{*}$, where $\mathbf{V}_{L}$ and $\mathbf{V}_{R}$ are unitary matrices and $\boldsymbol{\Sigma}$ is an ordered diagonal matrix $\left(\sigma_{i}>\sigma_{i+1}\right)$, it is shown [5] that if $\mathbf{F}$ is limited to be a unitary matrix, the optimum precoder is $\overline{\mathbf{V}}_{R}=\mathbf{V}_{R}(:, 1: M)$, i.e. a matrix constructed by the first $M$ columns of $\mathbf{V}_{R}$. Since for limited feedback systems, it is impossible to send back $\overline{\mathbf{V}}_{R}$, in [8] a set $(\mathcal{W})$ of suboptimum unitary matrices has been proposed.

The pairwise error probability (PEP) that vector $\mathbf{e}$ is detected instead of the transmitted vector $\mathrm{s}$ is

$$
\mathrm{P}(\mathbf{s} \rightarrow \mathbf{e} \mid \mathbf{H F})=Q\left(\sqrt{\|\mathbf{H F}(\mathbf{s}-\mathbf{e})\|^{2} \times \frac{\rho}{2 M}}\right) .
$$

By using the Rayleigh-Ritz inequality [9] we have

$$
\lambda_{\min }^{2}(\mathbf{H F})\|\mathbf{x}\|^{2} \leqslant\|\mathbf{H F} \mathbf{x}\|^{2} \leqslant \lambda_{\max }^{2}(\mathbf{H F})\|\mathbf{x}\|^{2}
$$

where $\mathbf{x}=\mathbf{s}-\mathbf{e}$, and $\lambda_{\min }$ and $\lambda_{\max }$ are the minimum and maximum singular values of $\mathbf{H F}$ respectively. Thus, for a given $\mathbf{H}$, the best selected precoder from $\mathcal{W}$ is obtained from

$$
\mathbf{W}_{\mathcal{I}}=\arg \max _{\mathbf{W}_{i} \in \mathcal{W}} \lambda_{\min }\left(\mathbf{H} \mathbf{W}_{i}\right)
$$

where the only feedback parameter is $\mathcal{I}$. By defining the distortion effect of suboptimal precoding as

$$
E_{\mathbf{H}}\left\{\lambda_{\min }\left(\mathbf{H} \overline{\mathbf{V}}_{R}\right)-\lambda_{\min }\left(\mathbf{H} \mathbf{W}_{i}\right)\right\}
$$

and using the properties of Grassmannian subspace packing, it is shown [8] that (5) is minimized when the minimum projection two-norm distance between any pair matrices of $\mathcal{W}$ is maximized. The projection two-norm distance is defined as

$$
\begin{aligned}
d_{\text {proj }}\left(\mathbf{W}_{i}, \mathbf{W}_{j}\right) & =\left\|\mathbf{W}_{i} \mathbf{W}_{i}^{*}-\mathbf{W}_{j} \mathbf{W}_{j}^{*}\right\|_{2} \\
& =\sqrt{1-\lambda_{\min }^{2}\left(\mathbf{W}_{i}^{*} \mathbf{W}_{j}\right)}
\end{aligned}
$$

Although several unitary matrix structures have been proposed in literature for differential unitary space-time modulation and precoding [10], in this paper, for simulations and comparisons, we use the unitary matrix structure proposed in [11] and optimized in [8] based on pairwise projection two-norm distance.

\section{Minimum Distance Based Precoding}

To improve the unitary precoding, we propose to use a general precoder rather than unitary one, for limited feedback systems. The precoder matrix $\mathbf{F}$, in general case can be written as $\mathbf{F}=\mathbf{U}_{L} \mathbf{A} \mathbf{U}_{R}^{*}$, where $\mathbf{U}_{L}$ and $\mathbf{U}_{R}$ are unitary matrices and $\mathbf{A}$ is a diagonal matrix. The optimal $\mathbf{U}_{L, o p t}=\overline{\mathbf{V}}_{R}$ is not available at the transmitter due to limited feedback channel. We propose to use $\mathbf{U}_{L}=\mathbf{W}_{\mathcal{I}} \tilde{\mathbf{U}}_{L}$, where $\mathbf{W}_{\mathcal{I}} \in \mathcal{C}^{N_{t} \times M}$ is obtained from (4) and $\tilde{\mathbf{U}}_{L} \in \mathcal{C}^{M \times M}$ is a unitary matrix. Therefore, the feedback parameters are $\mathcal{I}$ and parameters describing $\tilde{\mathbf{U}}_{L}, \mathbf{A}$ and $\mathbf{U}_{R}^{*}$, that are all $M \times M$ matrices. Since for $M>2$, the number of feedback parameters are too many but for $M=2$ are tolerable (it will be shown), and also this case seems to be the most practical case, we focus on finding the optimum precoder for $M=2$, while $N_{t}$ and $N_{r}$ are arbitrary and the routine is the same for higher dimensions.

\section{A. Channel Diagonalization}

When $\mathbf{W}_{\mathcal{I}}$ is determined (4), by considering the svd of $\mathbf{H} \mathbf{W}_{\mathcal{I}}=\mathbf{W}_{L} \mathbf{D} \mathbf{W}_{R}^{*}$ and since $\mathbf{W}_{L}$ is known to the receiver, we can multiply (1) by $\mathbf{W}_{L}^{*}$ to obtain

$$
\mathbf{y}=\sqrt{\rho} \mathbf{D} \mathbf{W}_{R}^{*} \tilde{\mathbf{U}}_{L} \mathbf{A} \mathbf{U}_{R}^{*} \mathbf{s}+\mathbf{z}
$$

where $\mathbf{z}$ has the same characteristics as $\mathbf{v}$ and the factor $1 / \sqrt{M}$ is absorbed in $\mathbf{A}$ as a power distributer matrix.

To determine the feedback matrices, we use the exact minimum distance of transmitted vectors:

$$
d_{\min }=\min _{\mathbf{x}}\left\|\mathbf{D} \mathbf{W}_{R}^{*} \tilde{\mathbf{U}}_{L} \mathbf{A} \mathbf{U}_{R}^{*} \mathbf{x}\right\|
$$

where $\mathbf{x}=\mathbf{s}-\mathbf{e}$ for all possible transmitted vectors. It is shown [6] that $d_{\min }$ is maximized when $\mathbf{W}_{R}^{*} \tilde{\mathbf{U}}_{L}$ is a diagonal matrix (clearly a diagonal unitary matrix).

Any $2 \times 2$ unitary matrix $\mathbf{U}$ can be parameterized as (see Appendix)

$$
\begin{aligned}
\mathbf{U} & =\mathbf{U}_{1} \mathbf{B U}_{2} \\
& =\left[\begin{array}{cc}
1 & 0 \\
0 & e^{-j \alpha_{12}}
\end{array}\right]\left[\begin{array}{cc}
b & \bar{b} \\
\bar{b} & -b
\end{array}\right]\left[\begin{array}{cc}
e^{j \alpha_{21}} & 0 \\
0 & e^{j \alpha_{22}}
\end{array}\right]
\end{aligned}
$$

where $b \in[0,1], \bar{b}=\sqrt{1-b^{2}}$ and $\alpha_{i j} \in[0,2 \pi]$. Therefore, $\mathbf{W}_{R}$ can be written as $\mathbf{W}_{R}=\mathbf{U}_{L 1} \mathbf{B}_{L} \mathbf{U}_{L 2}$. Clearly, $\mathbf{W}_{R}^{*} \tilde{\mathbf{U}}_{L}$ will be a diagonal matrix if

$$
\begin{aligned}
\tilde{\mathbf{U}}_{L} & =\mathbf{U}_{L 1} \mathbf{B}_{L} \\
& =\left[\begin{array}{cc}
1 & 0 \\
0 & e^{-j \alpha_{L}}
\end{array}\right]\left[\begin{array}{cc}
b_{L} & \bar{b}_{L} \\
\bar{b}_{L} & -b_{L}
\end{array}\right] .
\end{aligned}
$$

Since $\mathbf{U}_{L 1}$ and $\mathbf{B}_{L}$ are determined by $\alpha_{L}$ and $b_{L}$ respectively, the equivalent channel matrix is diagonalized by only two parameters. Now, if we assume $\mathbf{U}_{R}=\mathbf{U}_{R 1} \mathbf{B}_{R} \mathbf{U}_{R 2}$ then

$$
\begin{aligned}
d_{\text {min }} & =\min _{\mathbf{x}}\left\|\mathbf{D} \mathbf{U}_{L 2}^{*} \mathbf{A} \mathbf{U}_{R 2}^{*} \mathbf{B}_{R}^{*} \mathbf{U}_{R 1}^{*} \mathbf{x}\right\| \\
& =\min _{\mathbf{x}}\left\|\mathbf{D} \mathbf{A} \mathbf{B}_{R} \mathbf{U}_{R 1}^{*} \mathbf{x}\right\|
\end{aligned}
$$

since both $\mathbf{U}_{L 2}$ and $\mathbf{U}_{R 2}$ are unitary and diagonal. Therefore, the equivalent channel and precoder model can be summarized as follows

$$
\hat{\mathbf{s}}=\sqrt{\rho} \underbrace{\sqrt{\rho_{d}}\left[\begin{array}{cc}
d & 0 \\
0 & \bar{d}
\end{array}\right]}_{\mathbf{D}} \underbrace{\left[\begin{array}{cc}
a & 0 \\
0 & \bar{a}
\end{array}\right]}_{\mathbf{A}} \underbrace{\left[\begin{array}{cc}
b_{R} & \bar{b}_{R} \\
\bar{b}_{R} & -b_{R}
\end{array}\right]}_{\mathbf{B}_{R}} \underbrace{\left[\begin{array}{cc}
1 & 0 \\
0 & e^{j \alpha_{R}}
\end{array}\right]}_{\mathbf{U}_{R 1}^{*}} \mathbf{s}
$$

where $a, d \in[0,1], \rho_{d}=d_{1}^{2}+d_{2}^{2}, d=d_{1} / \sqrt{\rho_{d}}$, and $d_{1}$ and $d_{2}$ are diagonal terms of $\mathbf{D}$. Here $a$ is the only parameter of $\mathbf{A}$ 
to allocate the power between sub-channels so that the total transmitted power is $\rho$. To find the remaining parameters in (12), we should first choose the signal constellation and then for all possible transmitted vectors, we should maximize (11) by simulations or maximize the minimum distance between any pairs of $\hat{\mathbf{s}}$ (12) geometrically.

\section{B. Optimum Parameters for Binary Constellation}

In binary case, the transmitted vector $\mathbf{s}$ and consequently $\hat{\mathbf{s}}$ have four different points in the complex plane. It can be shown that the minimum distance between these four points is maximized when $a=1, b_{R}=1 / \sqrt{2}$ and $\alpha_{R}=$ $\pi / 2$, independent of the channel parameter $d$. Therefore, the feedback parameters are $\mathcal{I}, \alpha_{L}$ and $b_{L}$. The equivalent system model in (12) is reduced to

$$
\hat{\mathbf{s}}=\sqrt{\frac{\rho d_{1}^{2}}{2}}\left[\begin{array}{ll}
1 & j \\
0 & 0
\end{array}\right] \mathbf{s} .
$$

Eq. (13) shows clearly that for binary case, the equivalent optimum channel depends on $d_{1}$ which is the $\lambda_{\max }\left(\mathbf{H} \mathbf{W}_{\mathcal{I}}\right)$. Therefore, the optimum precoder should be selected by the following optimization problem rather than (4):

$$
\mathbf{W}_{\mathcal{I}}=\arg \max _{\mathbf{W}_{i} \in \mathcal{W}} \lambda_{\max }\left(\mathbf{H} \mathbf{W}_{i}\right) .
$$

\section{Optimum Parameters for QPSK Constellation}

When the transmitted symbols are from a QPSK constellation, there are $4^{2}$ transmitted vectors or equivalently $4^{2}$ precoded points $(\hat{\mathbf{s}})$ in the complex plane. It can be shown [6] that the precoder parameters depend on $d$. Therefore, the feedback parameters are $\mathcal{I}, \alpha_{L}, b_{L}$ and $d$. When $d \gtrsim 0.955$, the optimum parameters are $a=1, b_{R}=\sqrt{(3+\sqrt{3}) / 6}$ and $\alpha_{R}=\pi / 12$, independent of the channel parameter $d$. In this case, (12) is reduced to

$$
\hat{\mathbf{s}}=\sqrt{\rho d_{1}^{2}}\left[\begin{array}{cc}
\sqrt{\frac{3+\sqrt{3}}{6}} & \sqrt{\frac{3-\sqrt{3}}{6}} e^{j \frac{\pi}{12}} \\
0 & 0
\end{array}\right] \mathbf{s} .
$$

When $d \lesssim 0.955$, the optimum parameters are $b_{R}=1 / \sqrt{2}$, $\alpha_{R}=\pi / 4$ and

$$
a=\sqrt{\frac{1-d^{2}}{1-2(\sqrt{2}-1) d^{2}}} .
$$

and the equivalent system model will be

$$
\hat{\mathbf{s}}=\frac{\sqrt{\rho \rho_{d}}}{2}\left[\begin{array}{cc}
d a & 0 \\
0 & \bar{d} \bar{a}
\end{array}\right]\left[\begin{array}{cc}
\sqrt{2} & +(1+j) \\
\sqrt{2} & -(1+j)
\end{array}\right] \mathbf{s} .
$$

\section{Simulation Results AND COMPARISONS}

In this section, a MIMO system with $N_{t}=4$ transmit antennas, $N_{r}=3$ receive antennas and $M=2$ substreams for multiplexing is considered $(\operatorname{MIMO}(4,3,2))$ as an example to demonstrate the pros and cons of the proposed limited feedback precoder. A set of $2^{6}$ unitary precoders derived in [8] is used for simulations which requires 6 bits of feedback for $\mathcal{I}$. Since other precoding parameters are continuous, an appropriate quantizing method should be adopted to maximize the

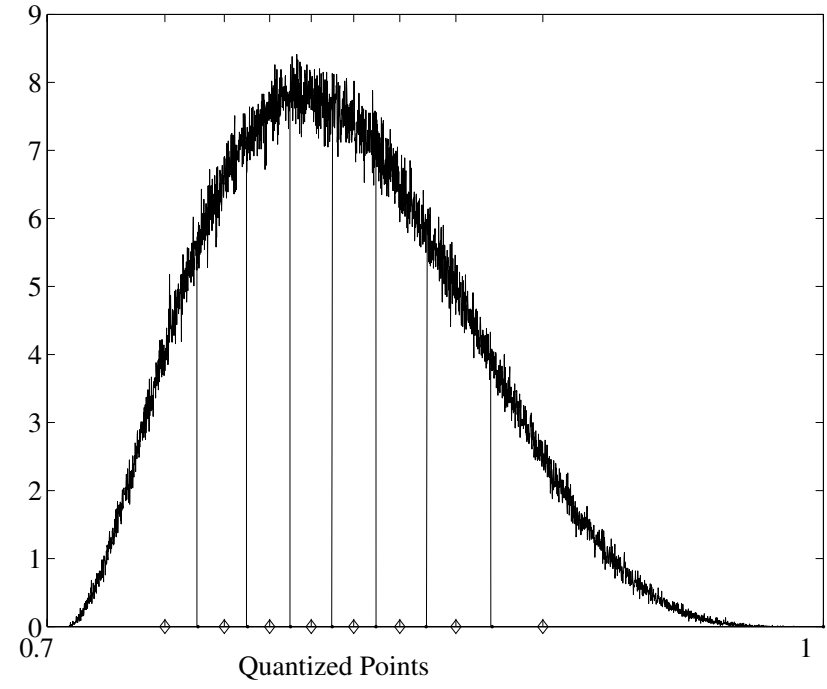

Fig. 1. The approximate pdf of feedback parameter ' $d$ '.

accuracy of the quantization and also the conveyed information per assigned bits.

\section{A. Quantization of Feedback Parameters}

Information theoric approach to quantization requires the quantized values to have the same probability of occurrence. Therefore, the first step for quantization is to evaluate the probability density function (pdf) of feedback parameters. The first feedback parameter is $\alpha_{L}$. Computer simulation shows that the pdf of $\alpha_{L}$ is uniform, $\mathcal{U}(0,2 \pi)$. Therefore, for a given $N_{b}$ bits of feedback for $\alpha_{L}$, the quantized index $i_{\alpha_{L}}$ and the regenerated value $\hat{\alpha}_{L}$ will be

$$
i_{\alpha_{L}}=\left\lceil\frac{\alpha_{L}}{\pi} 2^{N_{b}-1}\right\rceil \quad, \quad \hat{\alpha}_{L}=\pi \frac{2 i_{\alpha_{L}}-1}{2^{N_{b}}} .
$$

The pdf of $b_{L}$ obtained from simulation is

$$
f_{b_{L}}(x)=2 x \quad, \quad 0 \leqslant x \leqslant 1
$$

and consequently for a given $N_{b}$ bits of feedback for $b_{L}$, the quantized index $i_{b_{L}}$ and the regenerated value $\hat{b}_{L}$ will be

$$
i_{b_{L}}=\left\lceil b_{L}^{2} 2^{N_{b}}\right\rceil \quad, \quad \hat{b}_{L}=\sqrt{\frac{2 i_{b_{L}}-1}{2^{N_{b}+1}}} .
$$

Finally, Fig. 1 shows the pdf of feedback parameter $d$. The lines show the nonlinear quantization thresholds and the diamonds on the $x$-axis are the regenerated values $\hat{d}$ assigned to each region, for 3 bits of feedback for parameter $d$.

\section{B. Performance Comparisons}

Fig. 2 and Fig. 3 show the performance of $\operatorname{MIMO}(4,3,2)$ system with binary and QPSK signallings. In both figures, the performance of unitary precoding [8] with 6 bits of feedback for $\mathcal{I}$, the performance of optimal precoding with perfect knowledge of the channel at the transmitter [6] and the performance of our proposed method with the knowledge of exact feedback parameters, are included. Fig. 2 shows the performance of the proposed method in BPSK signalling when 


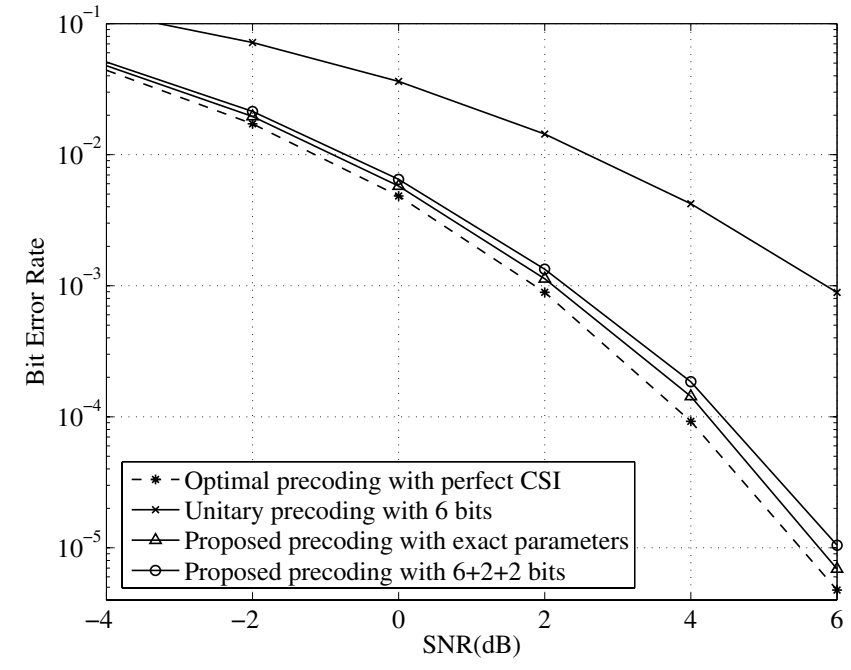

Fig. 2. Performance of binary signalling in $\operatorname{MIMO}(4,3,2)$ with different precoding techniques.

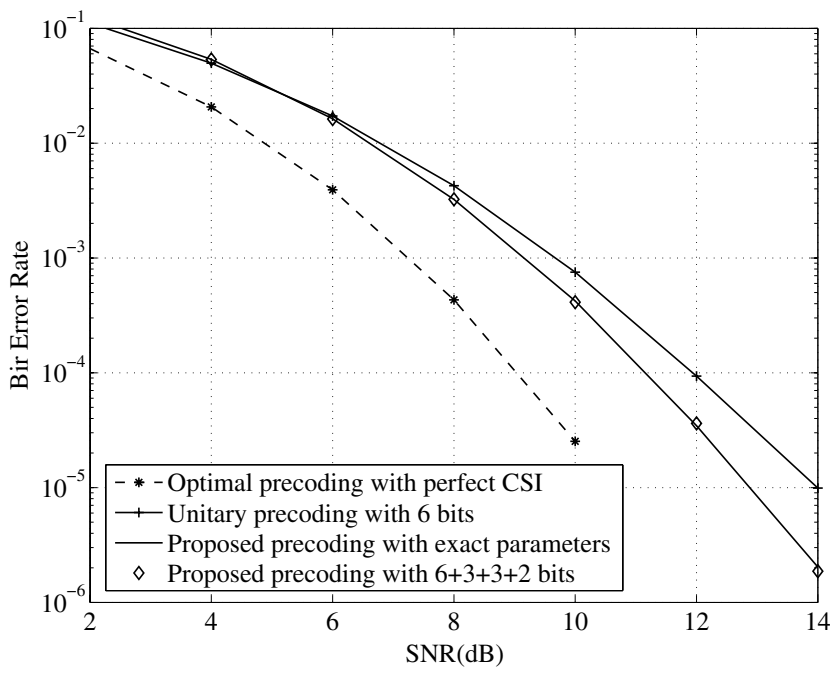

Fig. 3. Performance of QPSK signalling in $\operatorname{MIMO}(4,3,2)$ with different precoding techniques.

6,2 and 2 bits of feedback are assigned to $\mathcal{I}, \alpha_{L}$ and $b_{L}$ respectively. In Fig. 3, the assigned feedback bits are 6,3,3 and also 2 bits for parameter $d$. The results show that by adopting the proposed method which requires more bits of feedback, the performance of unitary precoding is improved, significantly for BPSK signalling. Also the figures show that the effect of quantization is relatively small.

\section{APPENDIX}

In general case, a $2 \times 2$ unitary matrix $\mathbf{U}$ can be written as

$$
\mathbf{U}=\left[\begin{array}{ll}
b_{11} e^{j \theta_{11}} & b_{12} e^{j \theta_{12}} \\
b_{21} e^{j \theta_{21}} & b_{22} e^{j \theta_{22}}
\end{array}\right]
$$

where $\theta_{i j} \in[0,2 \pi)$, and $b_{i j} \in[0,1]$ satisfy the following conditions:

$$
\begin{aligned}
& b_{11}^{2}+b_{21}^{2}=1 \\
& b_{21}^{2}+b_{22}^{2}=1
\end{aligned}
$$

By factorizing angles of the first row, we have

$$
\mathbf{U}=\left[\begin{array}{cc}
b_{11} & b_{12} \\
b_{21} e^{-j \theta_{1}} & b_{22} e^{j \theta_{2}}
\end{array}\right]\left[\begin{array}{cc}
e^{j \theta_{11}} & 0 \\
0 & e^{j \theta_{12}}
\end{array}\right]
$$

where $\theta_{1} \triangleq \theta_{11}-\theta_{21}$ and $\theta_{2} \triangleq \theta_{22}-\theta_{21}$. Now, by factorizing the angles of the first column we have

$$
\mathbf{U}=\underbrace{\left[\begin{array}{cc}
1 & 0 \\
0 & e^{-j \theta_{1}}
\end{array}\right]}_{\triangleq \mathbf{U}_{1}} \underbrace{\left[\begin{array}{cc}
b_{11} & b_{12} \\
b_{21} & b_{22} e^{j \theta}
\end{array}\right]}_{\triangleq \mathbf{B}} \underbrace{\left[\begin{array}{cc}
e^{j \theta_{21}} & 0 \\
0 & e^{j \theta_{22}}
\end{array}\right]}_{\triangleq \mathbf{U}_{2}}
$$

where $\theta \triangleq \theta_{2}+\theta_{1}$. Since $\mathbf{U}, \mathbf{U}_{1}$ and $\mathbf{U}_{2}$ are unitary matrices, the matrix $\mathbf{B}$ should be a unitary matrix, requiring to satisfy (A-1), (A-2) and the following condition:

$$
\begin{aligned}
b_{11} b_{12}+b_{21} b_{22} e^{j \theta} & =b_{11} b_{12}+b_{21} b_{22} \cos \theta+j b_{21} b_{22} \sin \theta \\
& =0
\end{aligned}
$$

(A-4) requires $b_{21} b_{22} \sin \theta=0$ which has four solutions. Three solutions related to $b_{21}=0, b_{22}=0$ and $\theta=0$ result in specific forms for unitary matrix $\mathbf{B}$. The acceptable solution is $\theta=\pi$ which requires

$$
\begin{aligned}
& b_{11}=b_{22} \triangleq b \quad, \quad b \in[0,1] \\
& b_{21}=b_{12}=\sqrt{1-b^{2}} .
\end{aligned}
$$

The final parametrization of $\mathbf{U}$ is presented in (9).

\section{REFERENCES}

[1] E. Telatar, "Capacity of multi-antenna Gaussian channels," AT\&T-BELL Labs, Tech. Rep., June 1995.

[2] B. Hassibi and B. M. Hochwald, "High-rate codes that are linear in space and time," IEEE Trans. Inform. Theory, vol. 48, pp. 1804-1824, July 2002.

[3] V. Tarokh, H. Jafarkhani, and A. Calderbank, "Space-time block codes from orthogonal designs," IEEE Trans. Inform. Theory, vol. 45, no. 5, pp. 1456 - 1467, July 1999.

[4] H. Sampath, P. Stoica, and A. Paulraj, "Generalized linear precoder and decoder design for MIMO channelsusing the weighted MMSE criterion," IEEE Trans. Commun., vol. 49, no. 12, pp. 2198-2206, 2001.

[5] A. Scaglione, P. Stoica, S. Barbarossa, G. Giannakis, and H. Sampath, "Optimal designs for space-time linear precoders and decoders," IEEE Trans. Signal Processing, vol. 50, no. 5, pp. 1051 - 1064, May 2002.

[6] L. Collin, O. Berder, P. Rostaing, and G. Burel, "Optimal minimum distance-based precoder for MIMO spatial multiplexing systems," IEEE Trans. Signal Processing, vol. 52, no. 3, pp. 617-627, 2004.

[7] P. Stoica and G. Ganesan, "Maximum-SNR spatial-temporal formatting designs for MIMO channels," IEEE Trans. Signal Processing, vol. 50, no. 12, pp. 3036-3042, 2002

[8] D. Love and R. J. Heath, "Limited feedback unitary precoding for spatial multiplexing systems," IEEE Trans. Inform. Theory, vol. 51, no. 8, pp. 2967-2976, 2005.

[9] R. A. Horn and C. R. Johnson, Matrix Analysis. Cambridge, U.K.: Cambridge Univ. Press, 1985.

[10] A. Ghaderipoor, M. Hajiaghayi, and C. Tellambura, "Unitary matrix design for differential space-time modulation and limited feedback precoding," IEEE International Symposium on Personal Indoor and Mobile Radio Communications (PIMRC), Helsinki, Finland, September 11-14, 2006.

[11] B. Hochwald and W. Sweldens, "Differential unitary space-time modulation," IEEE Trans. Commun., vol. 48, no. 12, pp. 2041 - 2052, Dec. 2000. 